\title{
Lösungsmöglichkeiten nach fehlgeschlagenen Osteosynthesen am Humerusschaft
}

\author{
Michael Paul Hahn
}

\section{Zusammenfassung}

Der Akzeptanzverlust der konservativen Verfahren hat zu einem Trendwechsel bei der Versorgung der Humerusschaftfrakturen geführt. Im Vordergrund stehen heute die Plattenosteosynthese und die Verriegelungsnagelung. Beide Verfahren haben typische Komplikationen, die jedem Operateur bekannt sein sollten. Für die Verriegelungsnagelung im Bereich des Humerusschaftes bestehen eindeutige Indikationsgrenzen, während die Plattenosteosynthese praktisch immer angewandt werden kann. Der Operateur ist gehalten, für die jeweilige Fraktursituation das am besten geeignete Verfahren auszuwählen. Bei Fehlschlägen mit der Verriegelungsnagelung ist ein Verfahrenswechsel auf eine Platte angezeigt. Dabei kann die Plattenosteosynthese jedoch nur dann alle Vorteile ausspielen, wenn die Regeln der AO beachtet werden. Als Mittel der Wahl bei fehlgeschlagenen Osteosynthesen muss die Plattenosteosynthese am Oberarm von allen Behandlern beherrscht werden.

\section{Einleitung}

Unter den Schaftbrüchen der langen Röhrenknochen nehmen die Humerusfrakturen eine Sonderstellung ein: Bei den Oberarmbrüchen überwiegen einfache Bruchformen im mittleren Schaftdrittel, der gut ausgebildete Weichteilmantel sorgt für reichlich Durchblutung und unterstützt die Retention bei funktioneller Behandlung, schwere Weichteilverletzungen oder offene Frakturen sind selten, selbst Achsabweichungen bis $20^{\circ}$ oder Verkürzungen sind für das funktionelle und kosmetische Ergebnis bedeutungslos.

Die konservative Behandlung war daher bislang die Methode der Wahl bei Humerusschaftfrakturen. Die bekannten Nachteile dieses Verfahrens haben die Entwicklung schonenderer Operationstechniken und besserer Implantate angeregt und damit einen Trendwechsel zur operativen Therapie eingeleitet $[2,7,8,14]$.

Der Vorteil der operativen Versorgung liegt in der kurzen Ruhigstellungszeit, der sofortigen Mobilisation der angren-

OP-JOURNAL 2004; 20: 188-194

(c) Georg Thieme Verlag KG Stuttgart · New York zenden Gelenke, der geringeren Strahlenbelastung durch entfallende Röntgenverlaufskontrollen und der kürzeren Zeit der Arbeitsunfähigkeit.

Gefürchtet bleiben die typischen Komplikationen der operativen Frakturversorgung. Dazu birgt jede operative Therapie das Risiko der fehlerhaften Osteosynthese. Typische Probleme und Lösungsmöglichkeiten sollen anhand von Fallbeispielen aufgezeigt werden.

\section{Epidemiologie und Klassifikation}

Bei den Schaftfrakturen des Humerus hat sich die AO/ASIF-Klassifikation im klinischen Alltag als praktikabel erwiesen und mittlerweile allgemein durchgesetzt [10]. Die Frakturen werden der Region 12 zugeordnet. Tytherleigh-Strong und Mitarbeiter untersuchten 249 Humerusschaftfrakturen [18]. Es fanden sich 63\% Typ-A-Frakturen, 26,2\% Typ-B- und 10,4\% Typ-C-Frakturen. 60\% der Frakturen lagen im mittleren Drittel der Diaphyse, $30 \%$ im proximalen und $10 \%$ im distalen Drittel.

Einfache Spiralfrakturen sind die häufigste Bruchform im proximalen, während im mittleren Schaftdrittel Quer- und kurze
Schrägbrüche überwiegen. Für das distale Drittel sind Typ-C-Frakturen typisch. Weniger als 10\% der Frakturen sind offene Frakturen. Die Humerusschaftfrakturen zeigen eine zweigipfelige Altersverteilung mit einem Gipfel im 3.Lebensjahrzehnt bei den Männern bei mittleren bis schweren Traumen und einem größeren Gipfel bei Frauen in der 7.Lebensdekade nach einem einfachen Sturz [18].

\section{Marknagelung}

Der Humerusschaft bietet aufgrund seiner trompetenförmigen Markraumform bei der reinen Marknagelung keine ausreichende Stabilität, so dass die Bildung von Pseudarthrosen vorprogrammiert ist [9]. Marknägel ohne Möglichkeit zur Verriegelung finden deshalb heute kaum noch Anwendung [15].

Bei fehlender Rotationsstabilität kommt es zur proximalen oder distalen Auslockerung des Nagels. Durch Biegebelastung kann das Implantat versagen (Abb.1a).

Die Therapie besteht in der Entfernung des Marknagels und anschließender Versorgung mit einer breiten, ausreichend langen Platte. Sollte dabei - insbesondere bei proximal lokalisierten Frakturen - die Verankerung der Platte problematisch sein, kann eine Augmentation mit Zement im Sinne einer Verbundosteosynthese erfolgen (Abb.1 b). Die zusätzliche Anlagerung von Spongiosa unterstützt die Frakturheilung. Bei proximal gelegenen Frakturen erfolgt die Implantation der Platte über einen anterioren Zugang (Abb.1 c).

\section{Bündelnagelung}

Bis zur Einführung der breiten AO-DCPlatte waren die geschlossene Marknagelung und die Bündelnagelung nach Hackethal die verbreitetsten Verfahren zur operativen Versorgung von Humerusschaftfrakturen $[4,6]$. 




Abb.1 (a) 52-jähriger Mann mit proximaler Humerusschaftfraktur rechts. Versorgung mit einem Universal-Marknagel ohne Verriegelung. 5 Jahre nach Unfall und Operation zeigt sich eine atrophe Pseudarthrose mit Verbiegung des Nagels. Lysesaum um den Nagel als Ausdruck der Instabilität. (b) Ausheilungsbild 2 Jahre nach Verfahrenswechsel (7 Jahre nach Unfall). Versorgung mit DC-Platte, Zement und Spongiosaplastik. Patient subjektiv beschwerdefrei. (c) Reizlose Narbe nach anteriorem Zugang zum proximalen Humerusschaft.
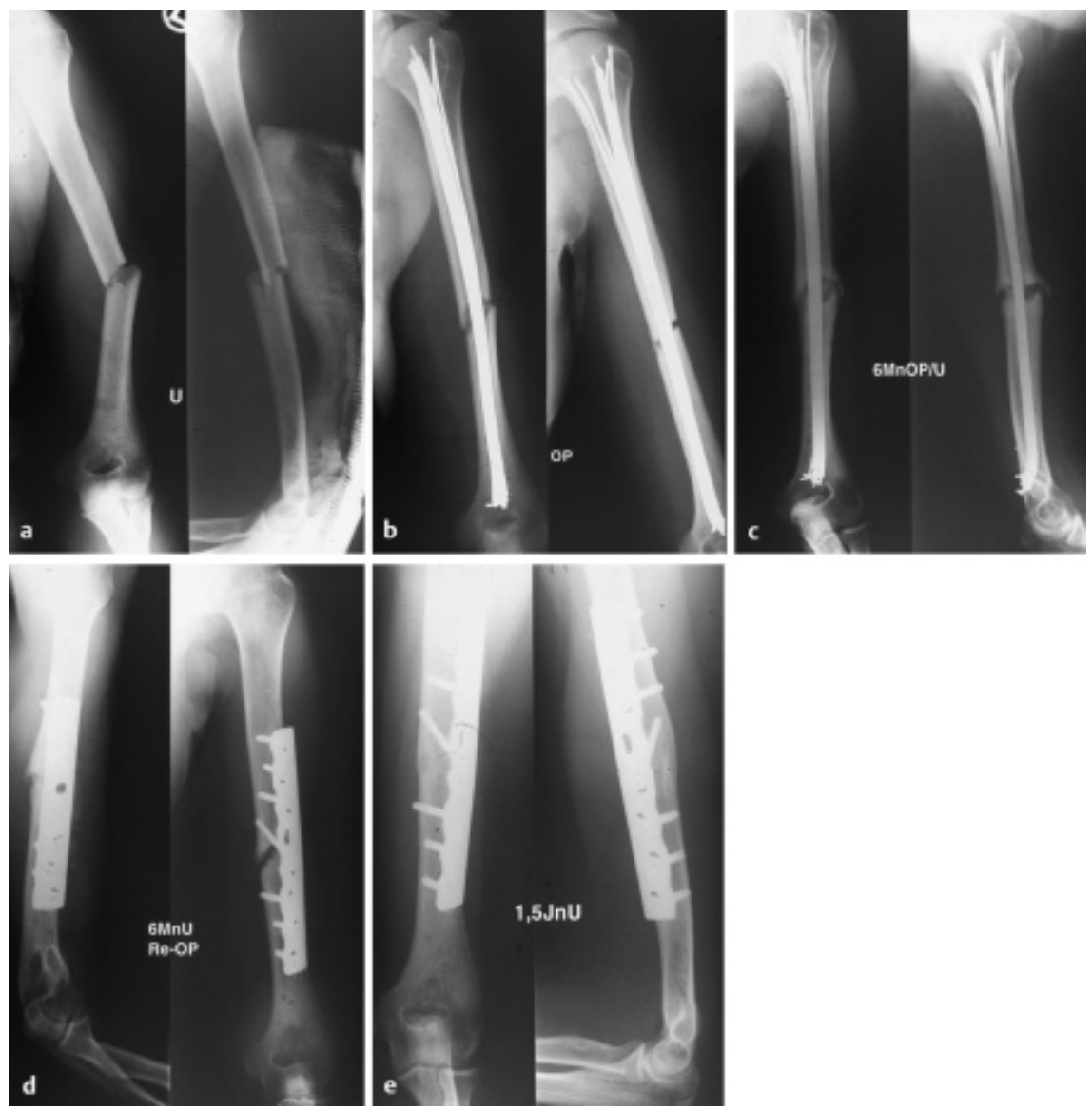

Abb. 2 (a) 72-jährige Patientin mit Querbruch im mittleren Drittel des Humerusschaftes. Initiale Versorgung mit „hanging cast“. (b) Versorgung der Fraktur mit zahlreichen flexiblen Markraumdrähten. Distraktion im Frakturbereich. (c) Ausbildung einer Pseudarthrose 6 Monate nach operativer Versorgung der Fraktur. (d) Verfahrenswechsel 6 Monate nach Unfall und Operation. Osteosynthese mit breiter 4,5 mm LC-DC-Platte und interfragmentärer Zugschraube. (e) Knöcherne Ausheilung der Fraktur 1,5 Jahre nach Unfall durch Kompression im Frakturspalt.
Diese Implantate waren für die Stabilisierung von Quer- und kurzen Schrägbrüchen geeignet. Bei der Bündelnagelung soll die Verankerung der gespreizten Nägel in der Spongiosa des Oberarmkopfes für eine ausreichende Distraktions- und Rotationsstabilität sorgen.

Die Bündelnagelung führt nur in der Hand des mit diesem Verfahren äußerst erfahrenen Operateurs zu guten Ergebnissen. Durch das Einführen mehrerer Drähte kann sehr leicht eine Distraktion im Frakturspalt auftreten (Abb.2au.b). Diese begünstigt eine zusätzliche Rotationsinstabilität mit konsekutiver Ausbildung einer Pseudarthrose (Abb. 2c).

Die Therapie der Distraktions- und Rotationsinstabilität besteht in der Versorgung der Fraktur mit einer breiten DCPlatte unter Kompression des Pseudarthrosenspaltes (Abb.2d u.e). Wenn immer möglich sollte eine interfragmentäre Zugschraube zur Erhöhung der Stabilität eingebracht werden. Auf eine ausreichende Länge der Platte ist zu achten. Die Möglichkeit zur minimalinvasiven Osteosynthese ist eingeschränkt, da in Schaftmitte der N.radialis immer dargestellt werden muss. Hier mag in Zukunft die endoskopischen Kontrolle weitere Fortschritte bringen [20].

\section{Intramedulläre Drahtung}

Kindliche Frakturen des Humerusschaftes können mit flexiblen intramedullären Titandrähten (sog. Prevot-Nägeln) operativ stabilisiert werden [13]. Dieses Verfahren sollte nicht auf den Knochen des Erwachsenen übertragen werden. Die immer wirksamen Rotationskräfte, die bei Kindern durch das kräftige Periost zu vernachlässigen sind, führen zu einer erhöhten Instabilität im Bereich der Fraktur, so dass die Frakturheilung wirksam behindert wird. Dadurch bilden sich regelmäBig Pseudarthrosen aus (Abb.3au.b). Die Therapie besteht wiederum in der stabilen langstreckigen Osteosynthese mit breiter AO-Platte.

\section{Seidel-Nagel}

Die Rotationssicherung ist bei intramedullärer Versorgung entscheidend. Diese lässt sich nur durch eine ausreichende proximale und distale Verblockung des Nagels gegenüber dem Knochen erreichen. 


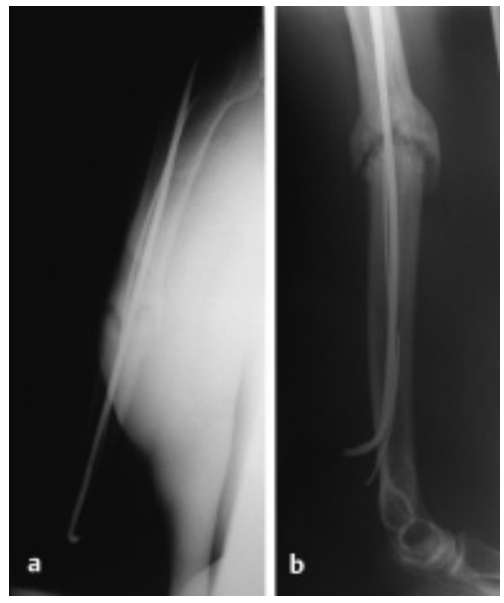

Abb. 3 (a) 46-jähriger Mann mit Humerusschaftquerfraktur im mittleren Drittel. Primärversorgung mit zwei flexiblen intramedullären Drähten. 4 Monate nach der Operation: In der Aufsicht gute Achsausrichtung. (b) In der Seitansicht: hypertrophe Pseudarthrose bei Instabilität im Frakturspalt. Ausgeprägte subjektive Beschwerdesymptomatik.
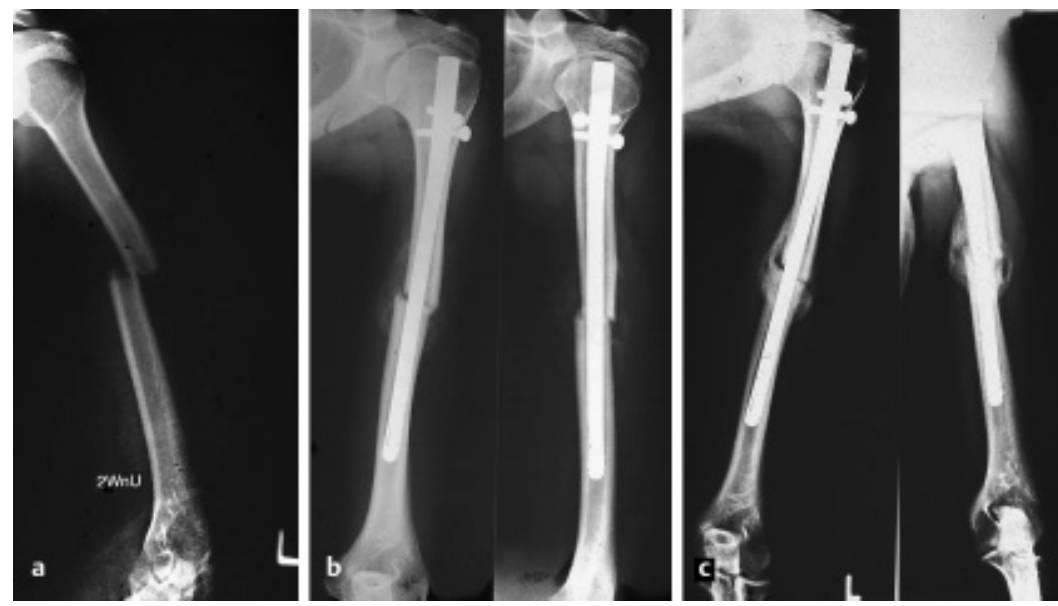

Abb.4 (a) 24-jährige Patientin mit Oberarmquerbruch im Übergang vom proximalen zum mittleren Drittel. 2 Wochen nach Unfall noch keine Zeichen von Kallusbildung. (b) 4 Wochen nach Versorgung der Fraktur mit einem Seidel-Nagel (6 Wochen nach Unfall). Beginnende Kallusbildung im Frakturbereich. (c) 3 Monate nach Operation zeigt sich eine insuffiziente Kallusüberbrückung bei Rotationsinstabilität aufgrund unzureichender distaler Verblockung des Seidel-Nagels im Markraumschaft.
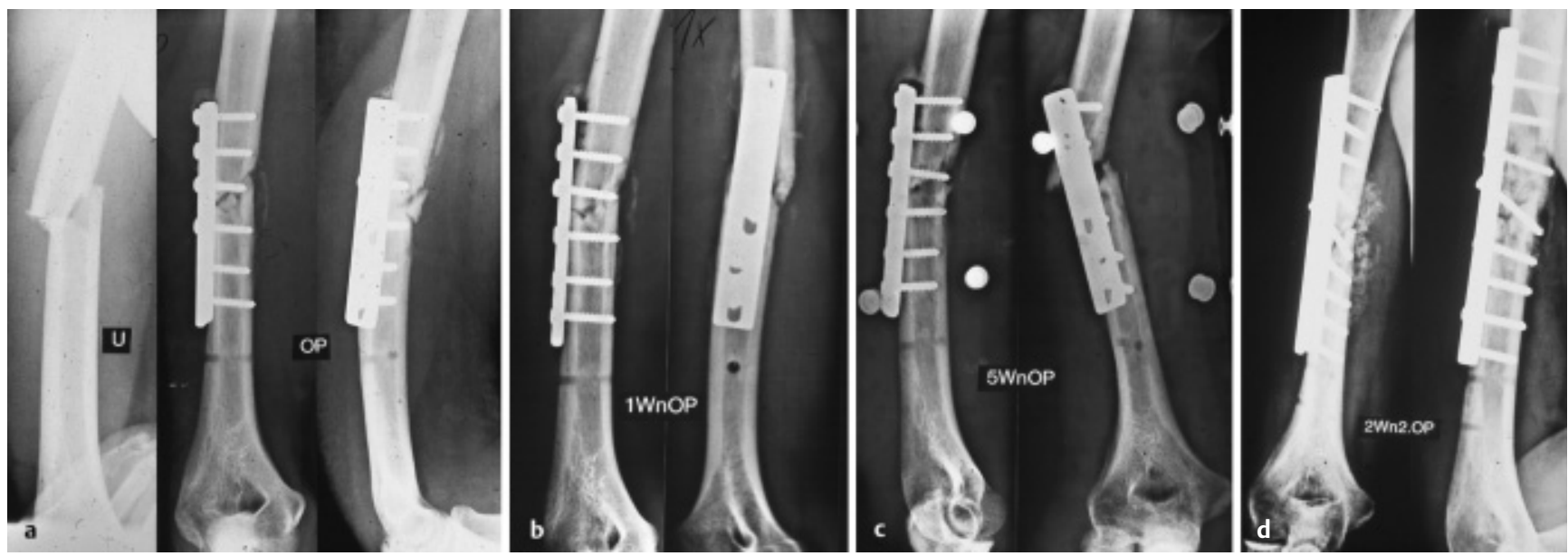

Abb.5 (a) 22-jähriger Patient mit Humerusquerfraktur Typ A1. Archivbild aus dem Jahr 1985. Versorgung mit kurzer Platte. (b) 1 Woche nach Operation: Ausriss der Platte aus dem proximalen Humerusschaft. (c) 5 Wochen nach OP: weitere Plattendislokation trotz externer Sicherung mit einem Brace. (d) 2 Wochen nach Zweiteingriff ( 7 Wochen nach Unfall): Osteosynthese mit deutlich längerer Platte und zusätzlicher Spongiosaplastik.
Der Seidel-Nagel realisiert die distale Verblockung durch einen Aufspreizmechanismus [15,17]. Eine ausreichende distale Rotationssicherung ist jedoch nur dann anzunehmen, wenn die Aufspreizung der Nagelspitze genügend weit distal erfolgt.

Dazu ist eine genau abgestimmte Nagellänge $\mathrm{zu}$ wählen, ohne dass es im Frakturspalt zur Distraktion kommt (Abb. 4a u.b). Bei relativer Rotationsinstabilität kann die Situation häufig durch eine vorübergehende externe Sicherung mit einem Brace gebessert werden. Die endgültige Frakturheilung verzögert sich in diesem Falle deutlich (Abb.4c). Bei anhaltender Instabilität ist die Indikation zum Verfahrenswechsel gegeben. Die Pseudarthrose kann durch eine Plattenosteosynthese zur Ausheilung gebracht werden.

\section{Plattenosteosynthese}

Bei Versorgung der Humerusschaftfraktur mit einer Platte dürfen die auftretenden Kräfte nicht unterschätzt werden. Es ist keinesfalls ausreichend, die Verankerung proximal mit nur zwei Kortikalisschrauben (4 Kortikales) durchzuführen (Abb.5a) [5,16,19]. Die Hebelkräfte des
Arms führen in diesen Fällen zu einer Auslockerung des Implantates (Abb.5b).

Die Versorgung mit einem Brace kann die weitere Implantatauslockerung nicht verhindern (Abb.5c). Bei eingetretener Plattenlockerung sind immer eine Revision und Reosteosynthese indiziert (Abb.5d). Die Lösung besteht wiederum in der Versorgung mit einer langen DCPlatte. Zusätzlich muss bei atrophen Pseudarthrosen Spongiosa angelagert werden.

Komplexe Frakturen des distalen Drittels erfordern eine sichere Überbrückungs- 


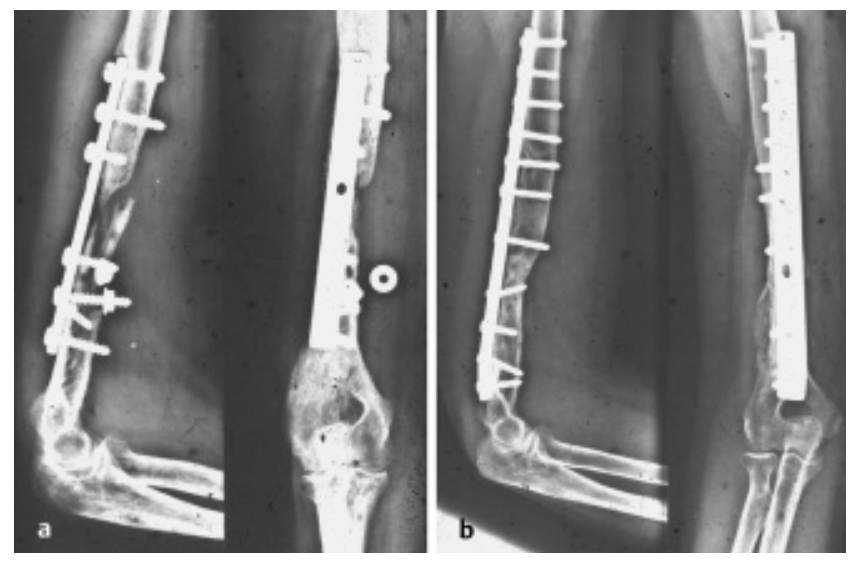

Abb.6 (a) Archivbild aus den 70er-Jahren: 47-jähriger Mann mit distaler Trümmerfraktur des Humerus Typ C, 7 Monate nach Unfall und Plattenosteosynthese mit Knochendefekt und avitalem Knochenfragment. (b) 15 Monate nach Zweiteingriff, 2 Jahre nach Unfall: knöcherne Heilung durch solide langstreckige Plattenosteosynthese und Spongiosaplastik.

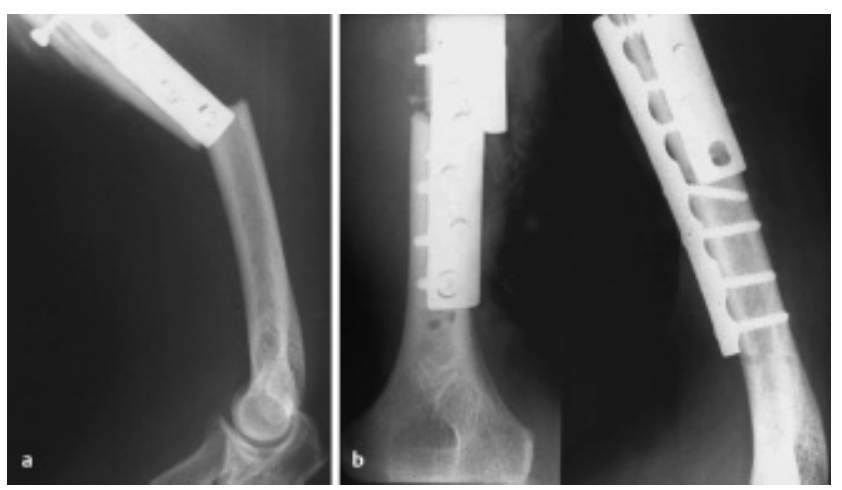

Abb.7 (a) 66-jähriger Patient mit Diaphysenfraktur des Humerus unmittelbar unterhalb einer von anterolateral eingebrachten Platte. Frakturverlauf durch das unterste Schraubenloch, (b) Osteosynthetische Versorgung mit zweiter Platte im Winkel von 90 Grad zur ersten. In diesem Falle Einbringen der Platte über einen ventralen Zugang.

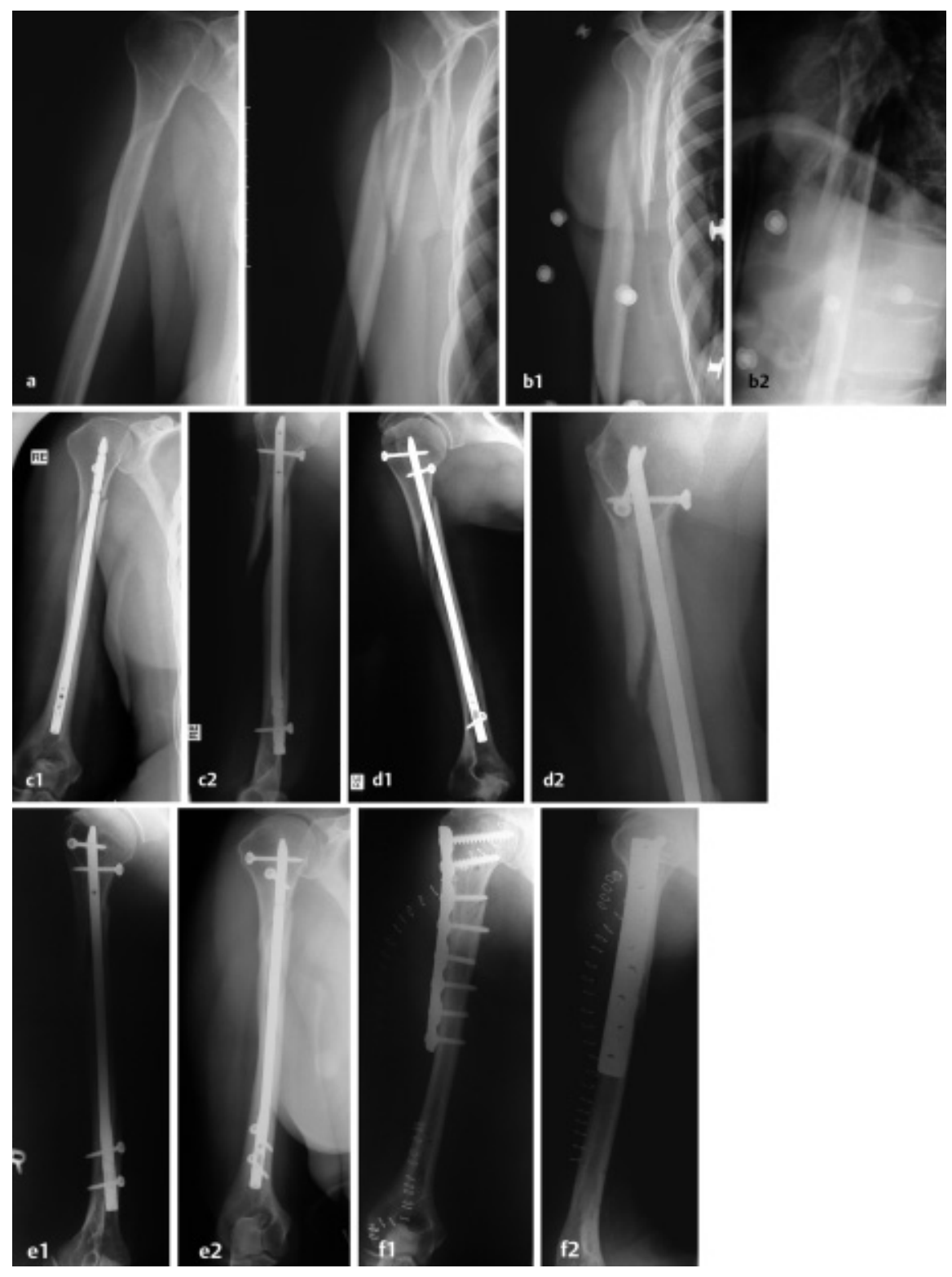

Abb. 8 (a) Unfallbild einer 65-jährigen Patientin mit proximalem Spiralbruch des Humerus. Indikationsstellung zur primär konservativen Behandlung. (b: 1,2) 2 Wochen nach Unfall: Deutliche Dislokation der Fraktur im Brace. (c: 1,2) Osteosynthese mit retrogradem Verriegelungsnagel. Nur je ein proximaler und distaler Bolzen zur Rotationssicherung. (d: 1,2) 4 Wochen nach Primäreingriff wird proximal ein weiterer Bolzen eingebracht. (e: 1,2) 6 Wochen nach Primäreingriff wird auch distal ein weiterer Bolzen eingebracht. (f: 1,2) 5 Monate nach Unfall klagt die Patientin weiter über Schmerzen und Instabilität mit Gebrauchsunfähigkeit des Arms. Wechsel auf eine breite DC-Platte zur Stabilisierung des proximalen Humerus bei ausgebliebener Frakturheilung. 



Abb.9 (a: 1,2) 57-jähriger Mann mit Humerusfraktur im Übergang vom mittleren zum distalen Schaftdrittel. 4 Wochen nach osteosynthetischer Versorgung mit UHN. In beiden Ebenen gute Achsausrichung. Distale Verriegelung mit einem Bolzen. Deutliche Lockerung des Bolzens. (b: 1,2) Detailaufnahme des gelockerten distalen Bolzens. Dadurch auch Dislokation der Schaftfraktur bei ausbleibender Frakturheilung. (c: 1 -3) Verfahrenswechsel auf langstreckige Platte mit Kompression im Frakturspalt.

osteosynthese. Schmale und kurze Platten sind den auftretenden Belastungen nicht gewachsen und führen regelmäßig zu Komplikationen (Abb.6a) [5,16,19]. Eine externe Stabilisierung mit einem Brace oder Cast ist aussichtslos. Erst durch Einbringen einer breiten DC-Platte und zusätzliche Spongiosaanlagerung kann in diesen Fällen die Fraktur zur Ausheilung gebracht werden (Abb.6b).

Entscheidend ist bei der Plattenosteosynthese die ausreichend lange Überbrückung der Fraktur, wobei nicht alle Schraubenlöcher besetzt werden müssen. Unbedingt ist eine Punktbelastung der Platte im Frakturbereich zu vermeiden, da diese unweigerlich zum Implantatversagen führt.
Frakturen des Humerusschaftes unterhalb des Plattenendes können analog einer periprothetischen Fraktur auftreten (Abb.7a). Diese Frakturen werden durch eine Kortikalisschwächung beim Einbringen der Plattenschrauben (dezentrale Lage der Schraube, häufiges Bohren) begünstigt. Da bei diesen Brüchen die konservative Therapie in den seltensten Fällen zur knöchernen Heilung führt, ist eine offene Einrichtung mit stabiler Fixation unumgänglich. Bei Frakturen im Übergangsbereich zum distalen Schaftdrittel wird die Osteosyntheseplatte entweder über einen dorsalen oder ventralen $\mathrm{Zu}$ gang eingebracht. Die von anterolateral eingebrachte Platte muss dabei nicht entfernt werden (Abb. 7 b). Nach knöcherner Heilung können dann bei Bedarf beide Platten wieder entfernt werden. Aufgrund der möglichen N.-radialis-Schädigung bei Metallentfernung ist die Indikation jedoch streng zu stellen.

\section{Ungebohrte Verriegelungsnägel}

Die ungebohrten Verriegelungsnägel für den Humerus haben die Vorherrschaft der Plattenosteosynthese aufgehoben $[1,2,11]$. Beide Verfahren sind mittlerweile als gleichwertig zu betrachten $[3,7,8,14]$. Die Verriegelungsnagelung fordert versierte Operateure und ist nicht für alle Frakturentitäten die bestmöglichste Therapieoption.

Proximale Schrägfrakturen sind für dieses Verfahren nur bedingt geeignet. Bei geschlossener retrograder Versorgung dieser Frakturen kann ein Muskelinterponat nicht sicher beseitigt werden. Damit fehlt häufig ein ausreichender Frakturkontakt $(\mathbf{A b b} .8 \mathbf{a}-\mathbf{c})$. Keinesfalls sollte die proximale Verriegelung mit nur einem Bolzen ausgeführt werden. Die resultierende relative Instabilität verhindert eine ausreichende Ruhe im Frakturspalt, so dass eine knöcherne Durchbauung - wenn überhaupt - nur sehr verzögert zu erzielen ist.

Auch durch proximale Verriegelung mit zwei Bolzen kann bei Schrägbrüchen eine Frakturheilung nicht immer sicher gewährleistet werden (Abb. 8d u.e).

Hier ist ein Verfahrenswechsel indiziert. Über einen anterioren Zugang wird eine breite Platte eingebracht. Dabei wird das Muskelinterponat beseitigt (Abb. 8f).

Das gleiche Phänomen ist auch bei unzureichender distaler Verriegelung zu erwarten. Aufgrund zu langer Hebelarme und des Spiels des Bolzen im Verriegelungsloch des Nagels kann keine ausreichende Stabilität im Frakturbereich erzielt werden (Abb.9au.b). Wiederum kann dieses Problem nur durch Verfahrenswechsel auf eine breite Platte behoben werden (Abb.9c).

Die im Bereich der unteren Gliedmaßen wirksamen Prinzipien der Dynamisierung können nicht ohne weiteres auf den Oberarm übertragen werden. Der Oberarm wird axial nicht belastet. Daher wird eine einseitige Aufhebung der Verriegelung nicht zu einer Verbesserung der Frakturheilung führen. Stattdessen resultiert - neben der zu erwartenden Rotationsinstabilität - durch das Gewicht des anhängenden Arms zusätzlich eine Distraktion im Frakturspalt (Abb. 10 a u.b). Bei ausbleibender Frakturheilung ist daher eine Kompression im Bruchspalt nötig. Diese wird durch einen Verfahrenswechsel auf eine Plattenosteosynthese erreicht (Abb.10c). 

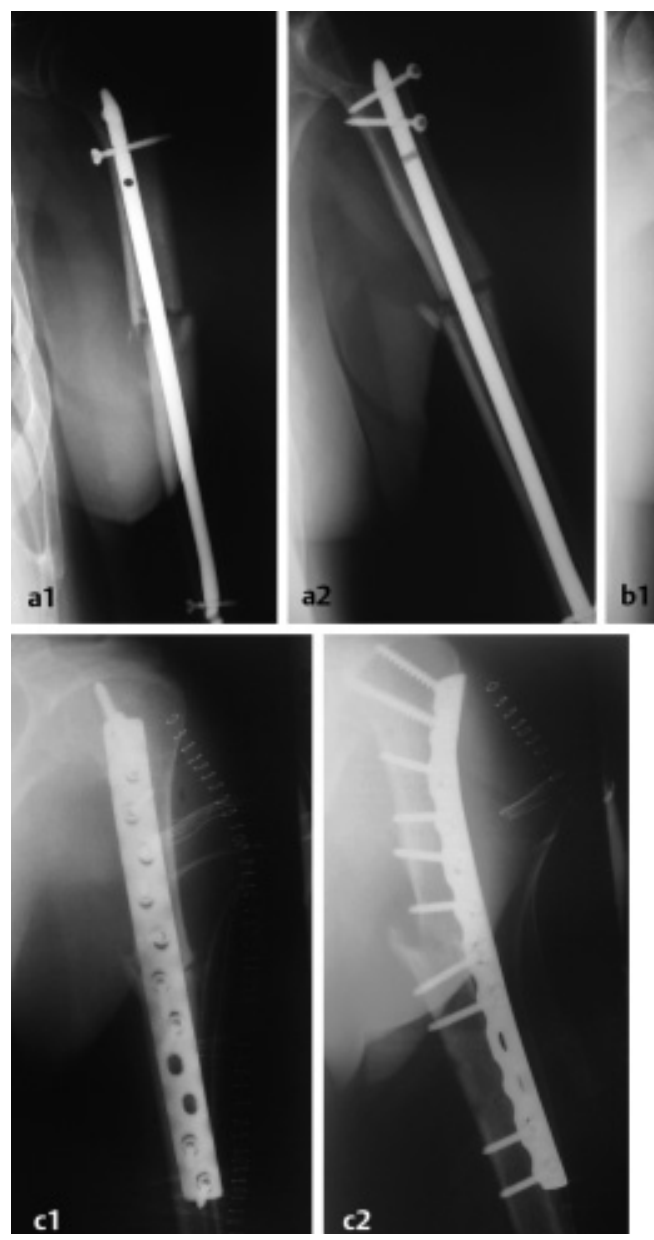

b1


Abb.10 (a: 1,2) 66-jähriger Patient mit proximaler Humerusschaftfraktur: 4 Wochen nach Operation. Distale Verriegelung mit einem Bolzen. Bei ausbleibender Frakturheilung wird die Indikation zur Dynamisierung durch Entfernung des distalen Bolzens gestellt. (b: 1,2) 5 Monate nach Dynamisierung hat sich durch Distraktion und Rotationsinstabilität eine Pseudarthrose ausgebildet. (c: 1,2) 7 Monate nach Erstoperation: Verfahrenswechsel auf lange Platte. Kompression im Pseudarthrosenspalt.

Langstreckige Schrägfrakturen des Humerus sind für eine Versorgung mit einem Humerusverriegelungsnagel weniger geeignet. Eine stabile Reposition lässt sich nur selten erreichen. Häufig kommt es zur Verkürzung des Humerus, was funktionell jedoch ohne Belang ist (Abb.11au.b). Problematischer ist die verzögerte knöcherne Heilung. Bleibt diese länger als 6 Monate aus, sollte eine zusätzliche Stabilisierung mit einer Platte erfolgen.

Trifft der von antegrad eingebrachte Nagel auf die Kortikalis oder lässt sich der von retrograd eingebrachte Nagel nicht mühelos in die Markraumhöhle vorschieben, kann es zu zusätzlichen Frakturen kommen (Abb.12a u.b). Lässt sich durch die Verriegelung die notwendige Stabilität nicht wiederherstellen, müssen weitere Maßnahmen ergriffen werden. Zunächst kann versucht werden, durch eine äußere Schienung den Oberarm ruhig zu stellen. Reicht dieses nicht aus, muss eine zusätzliche Plattenosteosynthese erfolgen.

\section{Diskussion}

Die konservative Behandlung von Oberarmschaftfrakturen hat in den letzten Jahren deutlich an Akzeptanz verloren und wird nur noch in bestimmten Situationen nach klar definierten Indikationen durchgeführt $[3,12,14]$.

Die operative Behandlung bietet den Vorteil der anatomischen Reposition und der sofortigen übungsstabilität. Vor allem bei polytraumatisierten Patienten und bei gelenknahen Frakturen wird dieser Vorteil evident.

In zunehmendem Maße konkurrieren bei der operativen Versorgung von Humerusschaftfrakturen die intramedullären Verfahren mit der Plattenosteosynthese $[1,2,7,8]$. Beide Therpieoptionen haben typische Komplikationen.

Die Plattenosteosynthese erfordert eine exakte Kenntnis der Anatomie und der Zugangswege. Nur bei subtiler Präparation und Erfahrung des Operateurs lassen sich neurologische Komplikationen vermeiden. Diese zählen nach wie vor zu den am meisten gefürchteten Problemen der Plattenosteosynthese $[3,14,20]$. Mini-
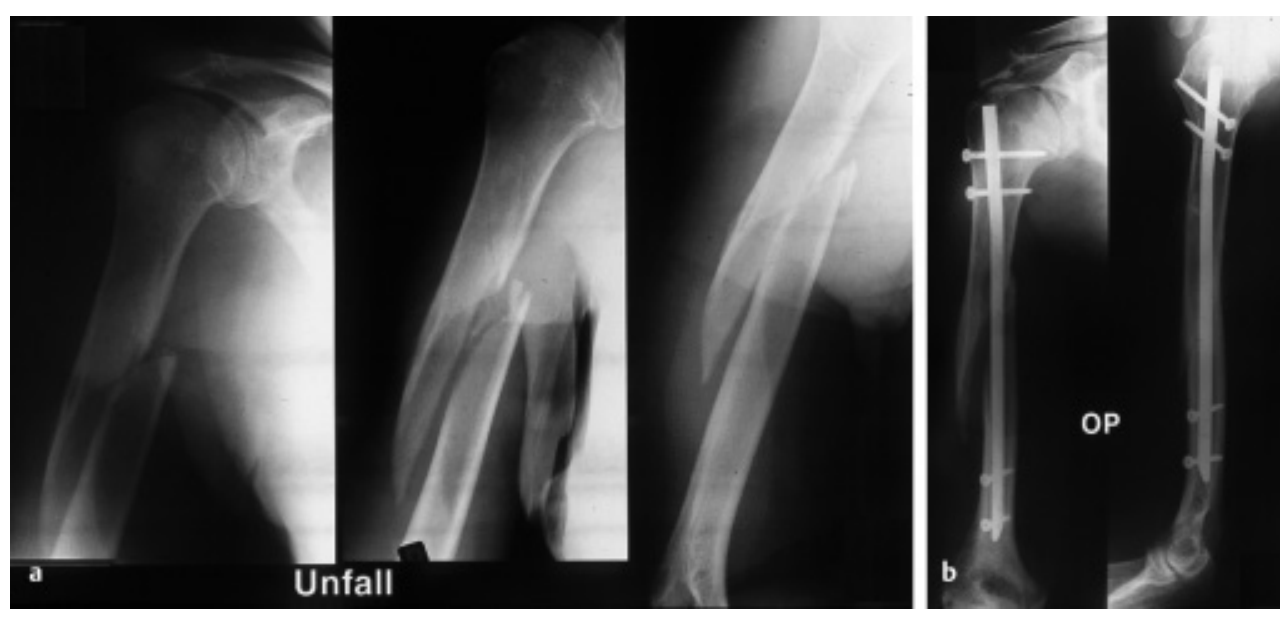

Abb.11 (a) Unfallbild eines 75jährigen Mannes mit proximaler Humerusschaftspiralfraktur.

(b) Implantation eines Verriegelungsnagels. Deutliche Verkürzung des Schaftes und nur marginaler Frakturkontakt. 

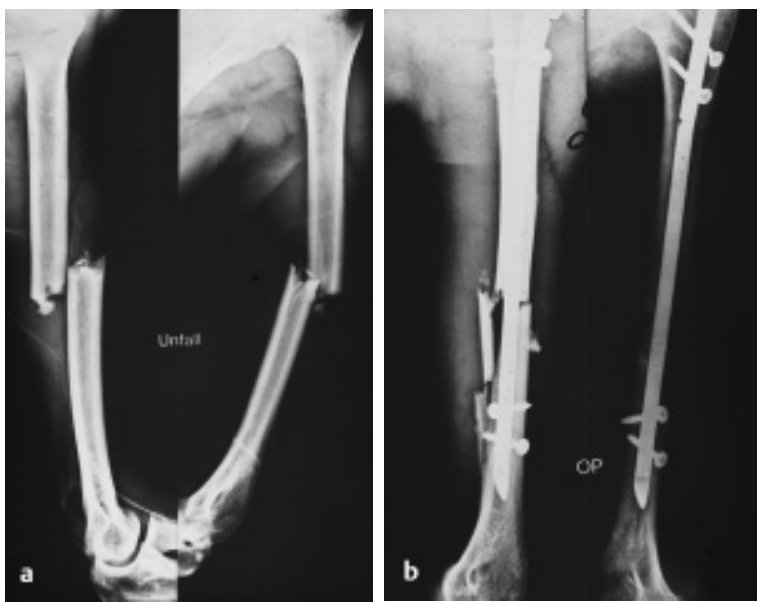

Abb.12 (a) Unfallbild eines 41-jährigen Mannes mit Querbruch des Humerus in Schaftmitte. (b) Osteosynthetische Versorgung mit antegradem Verriegelungsnagel (UHN). Beim Einschlagen des $\mathrm{Na}$ gels Aufsplitterung des distalen Humerus durch Auftreffen auf der Kortikalis.

malinvasive Vorgehensweisen haben bislang aufgrund der engen Beziehung des N. radialis zum Humerusschaft keine klinische Bedeutung erlangen können.

Die Platte muss im Verhältnis zum Knochen ausreichend dimensioniert sein. Am geeignetsten sind daher die breiten $4,5 \mathrm{~mm}$ AO-Platten (LC-DC-Platten). In Einzelfällen mag bei schmächtigen Knochen auch die schmale 4,5 mm Platte ausreichend sein [3]. Immer ist jedoch auf eine genügend weite Überbrückung der Fraktur zu achten. Werden diese Grundsätze nicht eingehalten, kommt es entweder zum Implantatversagen, Implantatbruch oder zur Ausbildung einer Pseudarthrose. Die Lösung dieser Probleme besteht in der Reosteosynthese mit einer adäquat dimensionierten Platte. Die Frakturheilung wird durch Spongiosaanlagerung unterstützt.

Die intramedullären Implantate der ersten Generation konnten sich nicht durchsetzen [3]. Die klassischen Marknägel können sich im Markraum des Oberarmschaftes nicht ausreichend verklemmen.

Der Seidel-Nagel war der erste Nagel mit proximaler und distaler Verriegelung $[15,17]$. Bei fehlender Verankerung der distalen Spreizschraube besteht jedoch auch bei diesem Nagel Rotationsinstabilität.

Dieses Problem tritt beim ungebohrten Verriegelungsnagel nicht auf $[1,2,7,11]$. Es bleibt die Gefahr der Schädigung des N.radialis bei geschlossener Nagelung, falls der Nerv im Bruchspalt interponiert ist.

Immer ist darauf zu achten, dass eine sichere Verblockung durch die eingebrachten Verriegelungsbolzen erreicht wird.
Da die Bolzen „Spiel“ in den Verriegelungslöchern der Implantate haben, müssen sie oftmals schräg eingebracht werden, um ausreichende Stabilität zu gewährleisten. Verriegelungen mit nur einem Bolzen sind in der Regel keinesfalls ausreichend.

Bei unzureichender Stabilisierung der Fraktur durch den Verriegelungsnagel ist eine konservative Behandlung mit einem Brace kaum die geeignete Lösung. Nur durch einen Wechsel auf die Plattenosteosynthese ist in diesen Fällen das Problem der Instabilität zu lösen $[3,5,8]$.

Die Plattenosteosynthese am Oberarm muss daher auch von den Chirurgen beherrscht werden, die bevorzugt intramedulläre Implantate einsetzen. Nur so können auftretende Probleme zielgerecht gelöst werden.

Angesichts der möglichen operationstechnischen Fehler und unter Berücksichtigung der jeweiligen Frakturcharakteristik sollte heute der Verriegelungsnagel immer dann Anwendung finden, wenn die Implantation möglich ist, während die Plattenosteosynthese durchgeführt wird, wenn sie nötig ist.

\section{Literatur}

${ }^{1}$ Blum J, Rommens PM, Janzing H, Langendorff HS. Retrograde Nagelung von Humerusschaftfrakturen mit dem UHN. Eine internationale multizentrische Studie. Unfallchirurg 1998; 101: $342-352$

${ }^{2}$ Fernandes FF, Matschke S, Hulsenbeck A Egenolf M, Wentzensen A. Five years' clinical experience with the unreamed humeral nail in the treatment of humeral shaft fractures. Injury 2004; 35: 264-271

${ }^{3}$ Gregory PR Jr. Fractures of the Shaft of the Humerus. In: R.W. Bucholz and J.D. Heckman (eds.): Rockwood and Green's Fractures in Adults. Lippincott Williams \& Wilkins, Philadelphia 2001, $5^{\text {th }}$ ed., Vol. 1, S. $973-997$

${ }^{4}$ Hackethal KH. Die Bündelnagelung. Springer Berlin Heidelberg 1961

${ }^{5}$ Hahn MP, Bayer-Helms H, Ostermann PAW, Muhr G. Indikationen und Ergebnisse der Plattenosteosynthese bei Humerusschaftfrakturen. Osteosynthese International 1996; 4: 246-252

${ }^{6}$ Hennig F, Link W, Wölfl R. Bündelnagelung eine Bilanz nach 27 Jahren. Akt. Traumatol. 1988; 18: $117-119$

7 Köstler W, Strohm PC, Südkamp NP. Neue Osteosyntheseverfahren am Humerus. Chirurg 2002; 73: 969-977

8 McCormack RG, Brien D, Buckley RE, McKee MD, Powell J, Schemitsch EH. Fixation of fractures of the shaft of the humerus by dynamic compression plate or intramedullary nail. J Bone Joint Surg Br 2000; 82: 336-339

${ }^{9}$ Mentzel E, Probst J, Wohlleben B. Oberarmschaftfrakturen und Oberarmschaftpseudarthrosen. Akt Traumatol 1982; 12: 229-234

${ }^{10}$ Müller ME, Nazarian S, Koch P, Schatzker J. The Comprehensive Classification of Fractures of the Long Bones. Springer, Berlin Heidelberg New York 1990

${ }^{11}$ Rommens PM, Verbruggen J, Broos PL. Retrograde Verriegelungsnagelung der Humerusfraktur. Eine klinische Studie. Unfallchirurg 1995; 98: 133-138

${ }^{12}$ Sarmiento A, Zagorski JB, Zych GA, Latta LL, Capps CA. Functional bracing for treatment of fractures of the humeral diaphysis. J Bone Joint Surg Am 2000; 82: 478-486

${ }^{13}$ Schmittenbecher PP, Blum J, David St, Knorr P, Marzi I, Schlickewei W, Schönecker G. Die Behandlung von Humerusschaftfrakturen und subkapitalen Humerusfrakturen im Kindesalter. Konsensusbericht der Sektion Kindertraumatologie der DGU. Unfallchirurg 2004; 107: 8-14

${ }^{14}$ Schittko A. Humerusschaftfrakturen. Chirurg 2004; 75: 833-847

${ }^{15}$ Schratz W, Wörsdörfer O, Klöckner C, Götze C. Behandlung der Oberarmschaftfraktur mit intramedullären Verfahren (Seidel-Nagel, Marchetti-Vicenzi-Nagel, Prevot-Pins). Unfallchirurg 1998; 101: 12-17

${ }^{16}$ Schweiberer L, Poeplau P, Gräber S. Plattenosteosynthese bei Oberarmschaftfrakturen. Sammelstudie der Deutschen Sektion der AO-International. Unfallheilkunde 1977 80: $231-235$

${ }^{17}$ Seidel H. Humeral locking nail: a preliminary report. Orthopedics 1989; 12: 219-226

${ }^{18}$ Tytherleigh-Strong G, Walls N, McQueen MM. The epidemiology of humeral shaft fractures. J Bone Joint Surg Br 1998; 80: $249-253$

${ }^{19}$ Winker H, Vosberg W, Cryis A. Behandlungsergebnisse nach Oberarmschaftfrakturen Aktuelle Traumatol 1993; 23 [Suppl 1]: $36-41$

${ }^{20}$ Zobrist R, Messmer P, Levin LS, Regazzoni P. Endoskopisch kontrollierte Stabilisation von Humerusschaftfrakturen. Unfallchirurg 2002; 105: 246-252

Prof. Dr. med. Michael Paul Hahn Ärztlicher Direktor

Klinik für Unfall- und Wiederherstellungschirurgie Klinikum Bremen-Mitte Sankt-Jürgen-Straße 1 D-28205 Bremen 